\title{
TRANSFORMACIONES Y APRENDIZAJES DE LAS RADIOS COMUNITARIAS EN ESPAÑA: HACIA UN MODELO DE RADIO INCLUSIVA
}

\section{Transformations and Learning of Community Radios in Spain: Towards an Inclusive Radio Model}

\section{Transformações e aprendizagens das rádios comunitárias na Espanha: a um modelo de rádio inclusiva}

\author{
García, Javier. Red de Investigación en Comunicación Comunitaria Alternativa \\ y Participativa (España) \\ javier@radioalmenara.net
}

Fecha de recibido: 1 de febrero de 2016

Fecha de aceptado: 18 de abril de 2016

\section{RESUMEN}

La historia de las radios comunitarias corre paralela al desarrollo de la democracia española. Estas emisoras fueron pioneras y se adelantaron a formas de comunicación y participación ciudadana que ahora, con Internet, resultan naturales: apropiación de las tecnologías, periodismo ciudadano, funcionamiento horizontal. Con este estudio se pretende mostrar cómo y hacia dónde se han producido las transformaciones de las radios comunitarias y cómo estas han pasado de ser un medio de difusión alternativo a configurar nuevas prácticas de comunicación comunitaria. Un aprendizaje que puede ser de gran utilidad para los nuevos medios y prácticas de comunicación en Internet.

Palabras clave: radio comunitaria, Tercer Sector de la Comunicación, inclusión, participación ciudadana. 


\section{ABSTRACT}

The history of the community radio runs parallel to the development of the Spanish democracy. These radio stations were pioneers and they advanced ways of communication and citizen participation that now, with the Internet, are ordinary: appropriation of the technology, citizen journalism and horizontal structure. With this study we want to show how and to where the transformation of community radio has been produced, and how these kind of projects have evolved from alternative media to new practices of community communication. A learning that can be of great utility for new media and communication processes on the Internet.

Keywords: community radio, third media sector, communication for social change, social innovation.

\section{RESUMO}

A história das rádios comunitárias corre paralelamente ao desenvolvimento da democracia espanhola. Estas emissoras foram pioneiras esse adiantaram a formas de comunicação e participação cidadã que agora com a internet resultam naturais: apropriação das tecnologias, jornalismo cidadão funcionamento horizontal. Com este estudo se pretende mostrar como e para onde se têm produzido as transformações das rádios comunitárias e como estas têm passado de ser um meio de difusão alternativo a configurar novas práticas de comunicação comunitária. Uma aprendizagem que pode ser de grande utilidade para os novos meios e práticas de comunicação na internet.

Palavras-chave: Rádio comunitária, terceiro setor da comunicação, inclusão, participação cidadã.

\section{Introducción}

El objetivo del presente artículo es mostrar la evolución y transformación de las radios comunitarias en España, desde su desarrollo en la década de los 80 hasta la actualidad. Si bien existe una amplia información relativa a experiencias de radio comunitaria, apenas encontramos algunos trabajos que sistematicen la experiencia española y profundicen en sus principales aportes. Por ese motivo, en el presente artículo se propone una sistematización de las distintas etapas y modelos de radios comunitarias que se han destacado en cada época, buscando clarificar y mejorar la comprensión del confuso y heterogéneo Tercer Sector de la Comunicación en España.

Para ello se realizó una revisión bibliográfica acerca de las radios comunitarias en España, junto con la observación y la participación como integrante de la Red de Medios Comunitarios (ReMC). De este análisis se pudo constatar la 


\section{DISERTACIONES}

ENSAYOS

Anuario electrónico de estudios en Comunicación Social

ISSN: 1856-9536

Doi: dx.doi.org/10.12804/revistas.urosario.edu.co/disertaciones/a.4535

Volumen 10, Número 1/ Enero-junio 2017

Versión PDF para imprimir desde

http://revistas.urosario.edu.co/index.php/disertaciones

existencia de distintas etapas, destacando como un hallazgo relevante la identificación del modelo de radio inclusiva como una práctica de comunicación comunitaria que se lleva a cabo en España desde los primeros años del siglo XXI.

\section{El Tercer Sector de la Comunicación y las radios comunitarias}

\section{Los medios impulsados por la ciudadanía y su diversidad}

En el ámbito de las organizaciones sin ánimo de lucro se ha popularizado la diferenciación en tres sectores: el sector público, el sector privado de índole mercantil, y el sector privado no lucrativo o tercer sector. Distinción que se ha aplicado al ámbito de la comunicación para referirse a las experiencias de carácter no lucrativo que no son público-estatales, ni privado-comerciales. Sin embargo, esta clasificación, surgida de una doble negación (no público, y no lucrativo), convierte al tercer sector en un espacio excesivamente heterogéneo, formado por actividades con más elementos diferenciales que comunes (García, 2013). Muchos medios no se sienten reflejados en la denominación de tercer sector porque no recoge sus especificidades y los agrupa con proyectos muy distintos. Por un lado, nos encontramos con la difícil conceptuación del tercer sector; por otro, nos encontramos con una multitud de términos para referirse a este tipo de medios de comunicación (comunitarios, alternativos, populares) y a sus distintas finalidades.

En la investigación La juventud española y los medios del Tercer Sector de la Comunicación, coordinada por el profesor Barranquero (2015), se profundiza en esta diversidad por medio de los datos aportados por los 94 medios de comunicación que participaron en el estudio. La información relativa a las misiones principales y las actividades desarrollas por los medios encuestados, nos permite agrupar el accionar de los medios del Tercer Sector en razón a tres finalidades principales: política o reivindicativa, social y educativa, y de ocio y cultura.

\section{Gráfico 1. Principales misiones y valores del medio}

Información independiente

Formación

Favorecer pluralismo

Educación y sensibilización

Acción social

Política reivindicativa

Animación cultural

Ocio y tiempo libre

Otros

Fuente: Barranquero y Villanueba, 2016. 


\section{DISERTACIONES}

ENSAYOS

Anuario electrónico de estudios en Comunicación Social

ISSN: 1856-9536

Doi: dx.doi.org/10.12804/revistas.urosario.edu.co/disertaciones/a.4535

Volumen 10, Número 1 / Enero-junio 2017

Versión PDF para imprimir desde

http://revistas.urosario.edu.co/index.php/disertaciones

\section{La radio comunitaria}

Si bien hay cierta tendencia a equiparar la expresión Tercer Sector de la Comunicación (TSC) con la de medios comunitarios o comunicación comunitaria, aquí pensamos que no se trata de términos equivalentes. Consideramos que las emisoras comunitarias forman parte del Tercer Sector que engloba un amplio espectro de otros medios.

El uso del término radio comunitaria no está exento de controversia y hay quienes prefieren usar otros (radio libre, social, alternativa, etc.) aunque se trata de un término definido y conceptualizado de forma más detallada y precisa que el de Tercer Sector de la Comunicación.

La Asociación Mundial de Radios Comunitarias (Amarc, 2010), define a las emisoras comunitarias como:

Actores privados que tienen una finalidad social y se caracterizan por ser gestionadas por organizaciones sociales de diverso tipo sin fines de lucro. Su característica fundamental es la participación de la comunidad tanto en la propiedad del medio, como en la programación, administración, operación, financiamiento y evaluación. Se trata de medios independientes y no gubernamentales, que no realizan proselitismo religioso ni son de propiedad o están controlados o vinculados a partidos políticos o empresas comerciales (p. 7).

Por su parte, el Relator Especial sobre la Libertad de Expresión de la ONU en su informe de 2010 se refiere a: Servicio de radio y prensa escrita no estatal de interés público operado por organizaciones, asociaciones o instituciones civiles y cualquier forma de organización de los pueblos indígenas sin carácter lucrativo y con finalidades educativas, culturales, populares al servicio del desarrollo de los diferentes sectores que conforman una comunidad de carácter territorial, etnolingüístico u otra, con intereses, retos compartidos y preocupaciones comunes, para mejorar la calidad de vida de sus congéneres y así encontrar un estado de bienestar para todos sus integrante (Mohammadiane y Muñoz, 2015, p. 328).

Según lo expuesto arriba, los elementos que diferencian y definen a la comunicación comunitaria con respecto a otras iniciativas de comunicación no lucrativas y no gubernamentales son: la diversidad respecto a la finalidad, el grupo o la organización que la promueve; su orientación a la mejora de la comunidad a la que se destina; y que la comunidad es la propietaria, gestora y la voz del medio.

Lo anterior no determina un medio de comunicación con unas características concretas, sino que presenta los principios que lo sustentan. Por tanto, existen múltiples configuraciones de emisoras comunitarias, que pueden tratarse como radio rural, urbana o destinada a un grupo concreto (comunidad de interés). Es la participación de la ciudadanía la que determina la finalidad y la funcionalidad de la radio comunitaria (Mohammadiane y Muñoz, 2015) donde la comunicación se concibe como un proceso horizontal, distinto de la verticalidad de la difusión de información en otros medios.

\section{Hitos de la experiencia española}

\section{El surgimiento de la radio reivindicativa}

Tras el final de la dictadura en España aparece un gran número de emisoras sin permiso para emitir que responden a la necesidad de expresión de distintos sectores y colectivos sociales. Este fenómeno de radios piratas comprende proyectos de diverso tipo: experimentales, comerciales independientes, municipales y de diversos sectores interesados 


\section{DISERTACIONES}

ENSAYOS

Anuario electrónico de estudios en Comunicación Social

ISSN: $1856-9536$

Doi: dx.doi.org/10.12804/revistas.urosario.edu.co/disertaciones/a.4535

Volumen 10, Número 1 / Enero-junio 2017

Versión PDF para imprimir desde

http://revistas.urosario.edu.co/index.php/disertaciones

en desarrollar la radio como un espacio de expresión política, cultural o educativa. "Después del franquismo y con la recuperación de las libertades democráticas se manifiesta una voluntad, podríamos decir incluso un placer, para el ejercicio de la libertad de expresión y comunicación" (Moragas, 1988, p. 92).

Entre la diversidad de radios existentes en la década de los 80 cobran gran protagonismo las denominadas radios libres, un fenómeno eminentemente urbano, que comienza a articularse mediante coordinadoras tanto de ámbito local como de tipo estatal. Su referente es el movimiento de radios libres surgido unos años antes en Francia e Italia (Bassets, 1981).

Para Martínez (1998), a partir del Manifiesto de Villaverde elaborado por la Coordinadora de Radios Libres en su encuentro de 1983, la definición de las radios libres se articula en cinco puntos:

1/ su carácter no profesional ni lucrativo; 2 / su funcionamiento autogestionario con toma directa de deci-

siones; 3 / su autonomía frente a grupos políticos, económicos y publicitarios; 4/ su participación al servi-

cio de la comunidad local; 5/ su lucha contra el monopolio y centralización de la comunicación (s/p).

Se trata de emisoras con un alto componente reivindicativo, vinculadas a organizaciones políticas donde sus protagonistas principales son activistas y militantes. Un ejemplo de esto es la implicación en la animación de la campaña y creación del debate contra la integración española en la OTAN, por eso el triunfo del Sí en el referéndum de 1986 va a significar un revés para este movimiento de emisoras (Serrano, 2009).

Las radios de este período posibilitaban el acceso de la ciudadanía a los medios de comunicación, a pesar que por su situación de semiclandestinidad estas radios limitaban su acceso al ocultar su ubicación o no tener un funcionamiento estable.

\section{La proliferación de la radio abierta}

La década de los 90 significa una etapa de recambio y reinvención. Algunos proyectos surgidos en los 80 continúan mientras ven nacer una nueva generación de radios. Una parte de los nuevos proyectos mantienen el espíritu reivindicativo de la primera etapa, mientras otros se orientan más como espacios de expresión juvenil. El término radios libres, de procedencia europea, empieza a conjugarse con el concepto de radios comunitarias de influencia latinoamericana y anglosajona. Hay radios que utilizan indistintamente ambos términos para definirse, mientras otras emisoras entienden que son dos tipologías diferentes.

Las administraciones dejan funcionar a estas emisoras. Los cierres y multas de la década anterior dan paso a una permisividad tácita, pero carente del reconocimiento legal. Algunas emisoras consiguen consolidarse y articular proyectos sólidos y logran acceder a subvenciones públicas que les permiten mejorar sus equipamientos e incorporar personal remunerado.

En esta etapa los protagonistas son los jóvenes del barrio y las distintas tribus urbanas que intercambian su música por medio de las ondas de radio. Las emisoras muestran una dimensión más cultural y de entretenimiento, frente a la acción política y reivindicativa que pierde protagonismo. Este modelo de radio abierta se configura como un espacio de libre acceso, donde cualquier persona puede realizar su propio programa de radio (convirtiendo a las radios en receptoras de propuestas) y pasa a formar parte de una asociación pagando una cuota que sirve para el sostenimiento del proyecto. 


\title{
DISERTACIONES
}

ENSAYOS

Anuario electrónico de estudios en Comunicación Social

ISSN: 1856-9536

Doi: dx.doi.org/10.12804/revistas.urosario.edu.co/disertaciones/a.4535

Volumen 10, Número 1 / Enero-junio 2017

Versión PDF para imprimir desde

http://revistas.urosario.edu.co/index.php/disertaciones

\section{Tiempos de intercambio de experiencias y creación de redes}

\begin{abstract}
El nuevo impulso que toma el movimiento de radios libres y comunitarias durante los 2000 s no se entiende sin el impulso del movimiento alterglobalización y el rol de las nuevas tecnologías en este. Internet se convierte en un importante aliado de las radios comunitarias, hay un resurgir de los espacios de encuentro e intercambio entre las radios y se sientan las bases que facilitarán la creación de nuevos espacios de coordinación. En esta etapa cobra otra vez protagonismo la acción política y la reivindicación social con el nacimiento de proyectos de prensa on line con la red Indymedia (Calle, 2005, p. 19).
\end{abstract}

En el ámbito de las radios se consolidan espacios como la Unión de Radios Libres y Comunitarias de Madrid (URCM) y en 2005 se crea la Red de Medios Comunitarios (ReMC) que promueve la celebración de encuentros anuales. También hay un desarrollo de las radios universitarias que tiene como hito en 2011 el nacimiento de la Asociación de Radios Universitarias (ARU). Los proyectos ya no se desarrollan de forma tan aislada como en épocas anteriores, hay mayor apoyo mutuo, intercambio de contenidos y realización conjunta. Las emisoras y personas más jóvenes toman referencia de las más experimentadas pero también aportan nuevos enfoques y saberes. Hay un proceso de formación y aprendizaje informal entre las distintas experiencias.

Si bien las emisoras comunitarias no han podido recuperar la incidencia y popularidad que lograron en los años 80 el sector muestra una maduración. Las radios han mejorado sus proyectos, su organización interna, su conexión con las organizaciones sociales y los mecanismos para la participación de la ciudadanía.

Así, mientras la crisis se lleva por delante muchas emisoras municipales históricas, en el ámbito de las radios libres y comunitarias se ha mantenido el número de proyectos (alrededor de un centenar), lo que muestra la sostenibilidad del sector, aunque quizá también, se debe a que se han "acostumbrado a sobrevivir entre constantes dificultades" (García, 2013, p. 123). Por otra parte, el movimiento $15 \mathrm{M}^{1}$ ha servido para reactivar el interés en el Tercer Sector de la Comunicación, poniendo en valor los proyectos históricos y posibilitando el surgimiento de nuevas iniciativas.

\section{Hacia una radio inclusiva}

\section{De la radio abierta a la radio inclusiva}

La incapacidad de la radiodifusión pública y comercial para satisfacer las necesidades de los grupos sociales marginados y en desventaja (Lewis, 2008) hace cada vez más relevante el rol que desempeñan los denominados medios comunitarios o del tercer sector en la construcción de diversos 'nosotros' desde abajo (Sáez, 2011). Es importante recordar que las radios comunitarias han sido espacios destacados en la participación y la expresión de la ciudadanía desde los inicios de la democracia. Además de servir de altavoz para distintos sectores y mostrar realidades invisibles en los medios convencionales, han asumido un importante rol de herramienta vertebradora de la comunidad en la que operan.

1 Movilización ciudadana surgida a partir de las protestas realizadas el 15 de mayo de 2011 que dan como origen el movimiento de indignados en España. 


\section{DISERTACIONES}

ENSAYOS

Anuario electrónico de estudios en Comunicación Social

ISSN: $1856-9536$

Doi: dx.doi.org/10.12804/revistas.urosario.edu.co/disertaciones/a.4535

Volumen 10, Número 1 / Enero-junio 2017

Versión PDF para imprimir desde

http://revistas.urosario.edu.co/index.php/disertaciones

Ante las dificultades para emitir y acceder a grandes audiencias, las radios comunitarias han desarrollado otras estrategias y formas de trabajo en el ámbito local. Con su política de puertas y micros abiertos han impulsado eventos comunitarios, colaborado con otras organizaciones de su territorio, y se han convertido en dinamizadoras de la vida social y cultural del barrio donde se ubican.

\section{La experiencia de las radios en los barrios de la periferia de Madrid}

Un buen ejemplo de las innovaciones producidas lo tenemos en la actividad que desarrollan las radios comunitarias de la periferia de Madrid. Durante su larga trayectoria han ido mutando, añadiendo nuevos repertorios pero manteniendo su carácter reivindicativo. En los años 90 desarrollaron un modelo de tipo radio abierta que permitió dar cabida a distintas demandas del barrio, como el acceso por parte de distintos grupos juveniles, a la colaboración con centros educativos y centros de servicios sociales. También se debe tener en cuenta la influencia de experiencias internacionales, en especial la actividad desarrollada por la Asociación Mundial de Radios Comunitarias (Amarc), que permite conocer experiencias inspiradoras como Radio La Colifata en el Hospital Psiquiátrico de Buenos Aires.

Las novedosas experiencias surgidas en los 90 son replicadas y proliferan, especialmente a partir del año 2000, como lo muestra la presencia de una quincena de programas realizados por personas con problemas de salud mental en las radios de Madrid (García y Meda, 2012). En la actualidad, la parrilla de estas emisoras cuenta con numerosos programas de inclusión social, realizados con diferentes colectivos como personas con discapacidad, jóvenes en riesgo de exclusión social, mayores, inmigrantes, etc. que se combinan con otros programas musicales, de información local o de opinión.

El hecho que las radios comunitarias contaran desde finales de los 90 con un espacio de coordinación como la Unión de Radios Libres y Comunitarias de Madrid (URCM) facilitó el intercambio de experiencias, metodologías y mecanismos para financiar los proyectos. Aunque no todas las radios de la URCM han incorporado la inclusión social con la misma intensidad, en un primer lugar tenemos a Radio Vallekas, Radio Enlace (Hortaleza), OMC Radio (Villaverde) y Radio Almenara (Tetuán), en las cuales una parte importante de sus programaciones son espacios realizados en colaboración con asociaciones y entidades sociales (García y Meda, 2012). Con menos recursos, ECO Leganés y Radio Ritmo (Getafe) también han incorporado este tipo de programas, mientras en los casos de Radio Fuga (Aranjuez) y Radio Cigüeña (Rivas) ha sido más puntual.

\section{La propagación del modelo de radio inclusiva}

Este modelo no se ha desarrollado exclusivamente en Madrid; sin embargo, es donde más se ha extendido. En este sentido, organizaciones de ámbito nacional como la Red de Medios Comunitarios (ReMC) y la Asociación de Radios Universitarias (ARU) han cumplido un papel importante en la propagación de proyectos de comunicación más inclusivos.

En Andalucía encontramos un número significativo de experiencias, entre las que destacan: Onda Palmeras, en Córdoba; Onda Color, en el barrio de Palma Palmilla de Málaga (Mohammadiane y Muñoz 2015); y UniRadio, la radio de la Universidad de Huelva, con programas como El Zapato Roto realizado por los internos del Centro Penitenciario de Huelva. Se trata así de proyectos que emergen en la última década fruto de una mayor planificación, 


\section{DISERTACIONES}

ENSAYOS

Anuario electrónico de estudios en Comunicación Social

ISSN: 1856-9536

Doi: dx.doi.org/10.12804/revistas.urosario.edu.co/disertaciones/a.4535

Volumen 10, Número 1 / Enero-junio 2017

Versión PDF para imprimir desde

http://revistas.urosario.edu.co/index.php/disertaciones

al contrario de lo que sucedió con las emisoras pioneras que iniciaron de forma más espontánea y que adquirió su aprendizaje a partir de la acumulación de experiencias.

En otras localidades es habitual que la programación de las radios comunitarias incluya algún espacio realizado por grupos de población tradicionalmente invisibilizadas o en riesgo de exclusión social. Pero también encontramos casos donde estas experiencias de radio se ha desarrollado al margen de las radios comunitarias o son desarrollados como una actividad interna de una entidad.

\section{Experiencias internacionales}

Este nuevo modelo que identificamos como radio inclusiva no es exclusivo de la experiencia española. También nos encontramos con numerosas propuestas similares en otros países, siendo su presencia más generalizada en países como Irlanda y Reino Unido, que cuentan con políticas públicas que configuran las radios comunitarias como herramientas de cohesión social.

Así, en la Ley de radio comunitaria del Reino Unido la clave es el aporte al "beneficio social" (Lewis y Mitchell, 2015, p. 253) de estos proyectos al destinarse a: audiencias no cubiertas por radio convencional, facilitar el debate y la expresión de opiniones, formación del voluntariado y para el empleo, fortalecimiento de la comunidad, promover la diversidad cultural y lingüística, fomentar la inclusión social y la participación ciudadana. A pesar de que también existen críticas al modelo inglés por propiciar la proliferación de entidades prestadoras de servicios de carácter social, sin vinculación al territorio y carentes de carácter reivindicativo, que ven en este sector un nicho para desarrollarse mediante la obtención de fondos públicos.

\section{Características del modelo de radio inclusiva}

Del análisis de las experiencias de las radios comunitarias españolas podemos extraer elementos comunes que nos permiten caracterizar un modelo específico de comunicación comunitaria al que hemos denominado como radio inclusiva:

- Convergen varias finalidades: se mantienen elementos de la radio reivindicativa (acción política) y de la radio abierta (cultura y entretenimiento) y se incorpora la acción social y el carácter educativo.

- Mayor diversidad y composición heterogénea en comparación con otros modelos en que los grupos son más homogéneos (activistas, jóvenes o grupos de afinidad).

- Incremento de la participación: la radio es tanto receptora como promotora de proyectos; cuenta con mecanismos de acogida, formación y articula vías de acceso para colectivos y grupos sociales que no tienen presencia en la emisora.

- Inclusión social: presencia y participación de colectivos que enfrentan barreras o dificultades para su participación en la comunidad.

- Participación en redes asociativas de su entorno y colaboración con otros agentes de la comunidad, tanto públicos como privados.

- Mayor complejidad del proyecto y necesidad de contar con personal remunerado o colaboradores especializados. 
En la bibliografía analizada no encontramos referencias a este modelo que hemos identificado, ni a sus peculiaridades respecto de otros modelos de comunicación comunitaria. Se ha optado por la denominación de radio inclusiva dado que esta da cabida a diversidad de sujetos y necesidades, incluyendo en su proyecto a colectivos especialmente excluidos del espacio público, siendo este su elemento más significativo frente a otras experiencias de radio comunitaria.

La configuración de este nuevo modelo puede ser considerada como un resultado de la superposición, dentro de un mismo proyecto, de las principales finalidades detectadas en el accionar de los medios del tercer sector de la comunicación. Aunque también podríamos considerar la radio inclusiva como una práctica de comunicación comunitaria en su máxima expresión, es decir, como un resultado natural de la labor de vertebración y dinamización de la comunidad, propio del actuar de las radios comunitarias.

\section{Figura 1. Distintos modelos de comunicación comunitaria}

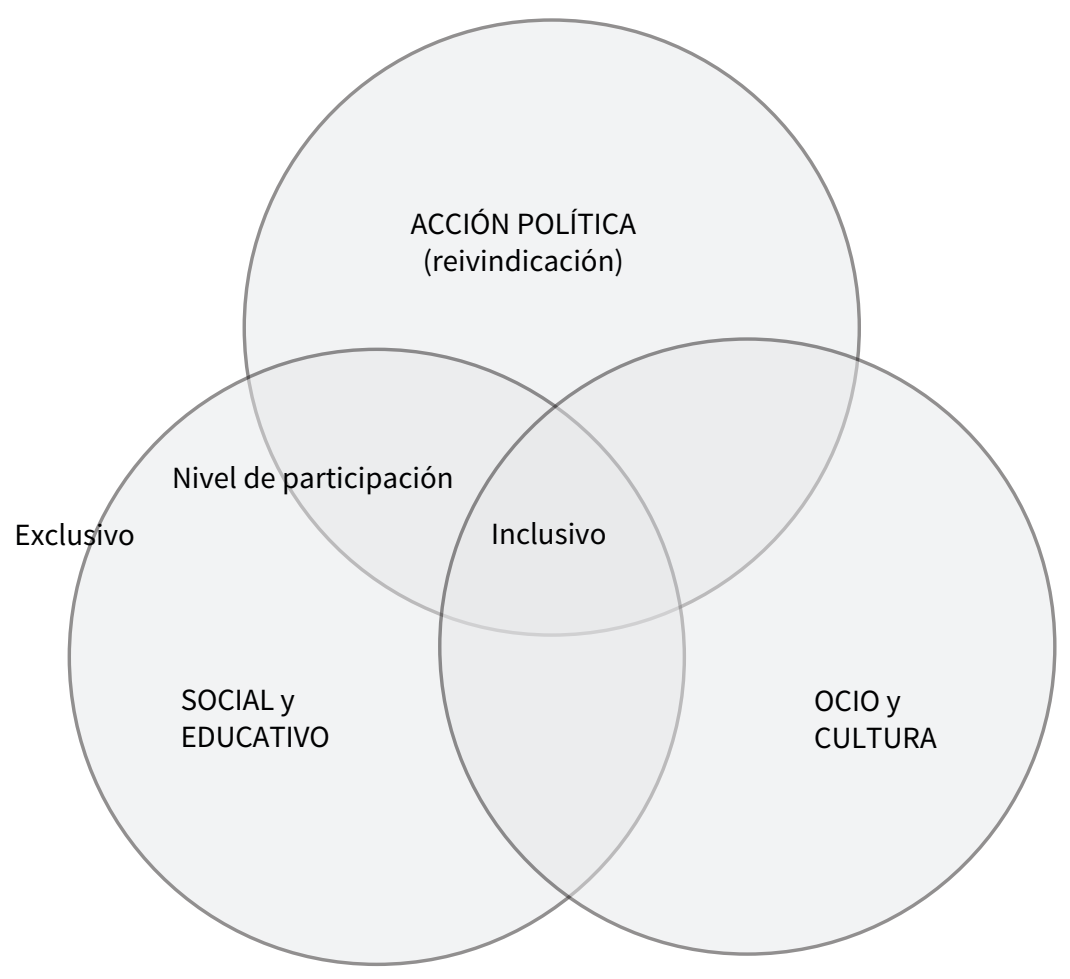

Fuente: elaboración propia.

\section{Desarrollo a contracorriente de la radio inclusiva}

A diferencia de otros países europeos en España no se habían desarrollado políticas públicas de promoción y apoyo al Tercer Sector de la Comunicación. Por el contrario, se había obstaculizado su existencia e impedido su acceso a licencias para emisión, razón por la cual tampoco existía financiación específica destinada a estas emisoras. 
Sin embargo, las radios comunitarias españolas han podido solventar estos obstáculos. Por un lado, han optado por emitir sin autorización enfrentándose a los poderes públicos, que terminaron por tolerar este atrevimiento. Por otra parte, las radios comunitarias, pese a su situación irregular, han podido acceder a fondos públicos gracias a algunos de los proyectos de carácter social que desarrollan. Paradójicamente, mientras los distintos gobiernos negaban el acceso al dial, las áreas de servicios sociales o juventud de las distintas Administraciones Públicas reconocían la labor desarrollada por las radios comunitarias y otorgaban subvenciones para el desarrollo de sus proyectos.

Tras décadas de reivindicaciones, con la Ley General de Comunicación Audiovisual (Ley 7 de 2010), se produjo el reconocimiento de estas emisoras, destacando su aporte social. El legislador español optó por la definición de "servicios de comunicación audiovisual comunitaria sin ánimo de lucro", cuyo objeto es atender las necesidades sociales, culturales y de comunicación específicas de comunidades y grupos sociales, así como fomentar la participación ciudadana y la vertebración del tejido asociativo. Para garantizar la existencia de estos servicios de comunicación se establece que la Administración General del Estado habilitará el dominio público radioeléctrico necesario para la prestación de estos servicios. Sin embargo, lo dispuesto en la ley no ha sido desarrollado y se mantienen los obstáculos al desarrollo de estas emisoras.

Así, a diferencia de lo ocurrido en países como Irlanda y Reino Unido, donde el Estado ha apoyado a las radios comunitarias promoviendo un modelo de radio inclusiva, en España han sido las propias emisoras comunitarias, en alianza con otras entidades y organismos sociales, quienes han tenido que inventarse un sistema de financiación.

En el caso español algunos de los elementos que han permitido la expansión del modelo de radio inclusiva han sido:

- Desarrollo del tercer sector con la consolidación y la maduración de proyectos y del grupo impulsor.

- Existencia de redes de coordinación y espacios de encuentro e intercambio entre los medios comunitarios.

- Aumento del número de mujeres en las radios comunitarias, lo cual ha contribuido a mejorar las prácticas, y a reorientar el enfoque de los proyectos. Según algunos estudios, las mujeres se vinculan a asociaciones por razones finalistas (ayudar a los demás) e instrumentales (defensa de derechos y opiniones) ${ }^{2}$ (Injuve, 2007).

- Acceso a financiación para proyectos de carácter social.

- Contar con personas formadas y con apoyo técnico (interno o externo).

\section{Conclusiones}

Los nuevos medios y plataformas de comunicación disponibles en Internet han superado a las radios comunitarias respecto a su capacidad de difusión, de acceso a la cultura y a contenidos alternativos. La radio de carácter local ha perdido vigencia como medio de difusión y corre el riesgo de convertirse en una herramienta obsoleta, sin capacidad de incidencia en su entorno.

2 Mientras los más jóvenes buscan en el asociacionismo espacios lúdicos y de ocio, según datos del Sondeo de Opinión 2007 (Injuve, 2007). 


\section{DISERTACIONES}

ENSAYOS

Anuario electrónico de estudios en Comunicación Social

ISSN: $1856-9536$

Doi: dx.doi.org/10.12804/revistas.urosario.edu.co/disertaciones/a.4535

Volumen 10, Número 1 / Enero-junio 2017

Versión PDF para imprimir desde

http://revistas.urosario.edu.co/index.php/disertaciones

Desde los 80 hasta la actualidad las radios comunitarias han dado respuesta a nuevos retos, adaptándose a las demandas sociales de cada época. Durante la transición democrática, las radios comunitarias fueron un vehículo para expandir el ejercicio de la libertad de expresión, dando cabida a reivindicaciones y corrientes políticas que no tenían presencia en los medios de comunicación públicos y privados. En esa primera etapa fueron protagonistas los activistas y los grupos políticos organizados, mientras que en los 90 lo fueron las oleadas de jóvenes de barrio que llegan a las radios comunitarias en busca de espacios donde expresarse y participar en la vida pública. Con la llegada de Internet y las redes sociales, tanto los activistas como los jóvenes han encontrado nuevos vehículos para comunicarse, lo que ha propiciado un menor interés hacia las radios comunitarias como medio de difusión.

En la última década las radios comunitarias han dado cabida a nuevas problemáticas y perfiles de participantes, siendo los grupos y colectivos que más sufren la exclusión, o con mayores dificultades en el ejercicio de sus derechos, los que muestran mayor interés en participar en las radios comunitarias. Estamos así ante un modelo que se configura de forma gradual y precaria ante la ausencia de políticas públicas destinadas a este tipo de proyectos. A esta situación se suma que: 1) el propio sector no ha identificado aún esta evolución, 2) tiende a mantener modelos de las etapas anteriores, o 3) encuentra dificultades para dar cabida a estos nuevos grupos por falta de conocimientos o recursos.

Las experiencias de radio inclusiva identificadas muestran la vigencia e importancia de las emisoras comunitarias, su potencia como herramienta de vertebración y su valiosa aportación a la inclusión social. Pero, a su vez, la ausencia de las condiciones que permitan una mayor expansión del modelo de radio inclusiva lleva a que estos grupos emergentes canalicen sus demandas mediante otros medios o decidan crear los suyos propios (blogs o radios online). Este cambio de medio puede afectar las transformaciones personales y grupales que no se dan con la misma intensidad en los espacios virtuales dada la naturaleza territorial, presencial y vivencial de los procesos colectivos que caracterizan la comunicación comunitaria. Todos estos son aspectos diferenciales que, junto con la acumulación de saberes durante su trayectoria, pueden ser explotados por las radios comunitarias para no convertirse en una especie en vías de extinción.

\section{Referencias}

1. Amarc. (2010). Principios para garantizar la diversidad y el pluralismo en la radiodifusión y los servicios de comunicación audiovisual. Recuperado de http://www.amarc.org/documents/40_Principios_diversidad_pluralismo.pdf

2. Barranquero, A. y Villanueva, S. (2016). Fisonomía, participación y pluralismo en los medios del tercer sector. En A. Barranquero (coord.) La juventud española y los medios del Tercer Sector de la Comunicación. Recuperado de https://jovenesytercersector.files.wordpress.com/2016/01/juventud_tercer_sector_comunicacion.pdf

3. Bassets, L. (1981). De las ondas rojas a las radios libres. Barcelona: Gustavo Gili.

4. Calle, A. (2005). Nuevos movimientos globales. Hacia la radicalidad democrática. Madrid: Popular.

5. García, J. (2013). Transformaciones en el tercer sector: el caso de las radios comunitarias en España. adComunica. Revista Científica de Estrategias, Tendencias e Innovación en Comunicación, (5), 111-131. doi: doi. org/10.6035/2174-0992.2013.5.8 
6. García, J. y Meda, M. (2012). ¡Qué locura de radio! Radios comunitarias y salud mental en España. En M. Martínez, C. Mayugo y A. Tamarit. (eds.), Comunidad y comunicación. Prácticas comunicativas y medios comunitarios en Europa y América Latina. (pp. 309-322). Madrid: Fragua.

7. Injuve. (2007). Sondeo 2007. Valores y actitudes, participación asociativa. Recuperado de http://www. injuve.es/sites/default/files/Sondeo2007-1a.pdf

8. Lewis, P. (2008). Promoting Social Cohesion: the Role of Community Media. Informe para el Consejo de Europa. Recuperado de https://rm.coe.int/CoERMPublicCommonSearchServices/DisplayDCTMContent?documentld $=0900001680483$ b32

9. Lewis, P y Mitchell, C. (2015). Fomento de la cohesión social: el papel de los medios de comunicación comunitarios. En M, Chaparro. (ed.), Medios de proximidad: participación social y políticas públicas. (pp. 249-269). Málaga: Luces de Gálibo.

10. Martínez, M. (1998). Islas de autogestión en un mar de contradicciones. Cuatro movimientos sociales alternativos en el Estado español (1978-1998). Recuperado de http://www.hartza.com/cabuenes.htm

11. Mohammadiane, A. y Muñoz, L. (2015). Las radios comunitarias andaluzas, una herramienta para la democratización de la comunicación. En M. Chaparro. (coord.), Medios de proximidad: participación social y políticas públicas. (pp. 327-345). Málaga: Luces de Gálibo.

12. Moragas, M. (1988). Espais de comunicació: experiéncies i perspectives a Catalunya. Barcelona: Ediciones 62.

13. Sáez, Ch. (2011). Lo público más allá de lo institucional: identidad, diversidad y Tercer Sector de la Comunicación. En B. León. (ed.), La televisión pública a examen. (pp. 174-185). Zamora: Comunicación Social Ediciones y Publicaciones.

14. Serrano, J. (2009). Radios Libres... toda una historia por delante. ZGZ Rebelde. Recuperado de http://www. zaragozarebelde.org/historia_radios_libres 